Rechtsmedizin 2022 · 32:481-488 https://doi.org/10.1007/s00194-022-00560-6 Angenommen: 21. Dezember 2021 Online publiziert: 15. Februar 2022 (c) Der/die Autor(en) 2022

\section{Aktuelle Trends des Cannabiskonsums}

\author{
J. Schürenkamp \\ Institut für Rechtsmedizin, Forensische Toxikologie, Universitätsklinikum Münster, Münster, Deutschland
}

Cannabis (Hanf) ist die am häufigsten konsumierte illegale Droge weltweit. Mit der Änderung des Betäubungsmittelgesetzes (BtMG) im Jahr 2017, das die Verschreibung von Cannabisblüten und Cannabisextrakten für medizinische Zwecke ermöglicht, sind die Verordnungszahlen stark angestiegen. Zudem entsteht neben dem seit Langem vorhandenen Konsum hanfhaltiger Lebensmittel bzw. Nahrungsergänzungsmittel ein Trend hin zu cannabidiolhaltigen Hanfölen oder Cannabidiolhanf. Konsequenz dieser Entwicklung sind verschiedenste Einlassungen zur Erklärung positiver Cannabinoidbefunde durch fraglich legale Cannabisprodukte. Daher ist die Kenntnis über diese verschiedenen Produkte und deren rechtliche Einordnung für die forensische Toxikologie von herausragender Relevanz. Ziel der Arbeit ist es, einen Überblick über aktuelle Trends des Cannabiskonsums zu geben und, ausgehend von der Cannabispflanze, die verschiedenen Cannabisprodukte mit ihren Inhaltsstoffen, ihrer Dosierung und ihren Indikationsgebieten darzustellen sowie diese rechtlich einzuordnen.

\section{Die Cannabispflanze und ihre Inhaltsstoffe}

Cannabis ist eine bereits seit dem 6. Jahrtausend v. Chr. bekannte Pflanzengattung aus der Familie der Cannabaceae (Hanfgewächse). Ob es sich bei der Cannabispflanze um eine monotypische, aber sehr variable Gattung Cannabis sativa handelt oder verschiedene Arten (Cannabis sativa, Cannabis indica, Cannabis ruderalis und Cannabis afghanica) mit unterschiedlichen geografischen, chemotypischen und morphologischen Merkmalen unterschieden werden können, ist in der Literatur noch immer umstritten [1].
Cannabis ist eine einjährige, zweihäusige (diözische), blühende, krautige Pflanze, in deren Drüsenhaaren insbesondere auf der Blütenoberfläche weiblicher Pflanzen eine cannabinoidreiche Terpenoidsekretion stattfindet. Zur Steigerung der Cannabinoidmenge werden weibliche Klone bei optimierten Lichtverhältnissen und Nährstoff- sowie Bewässerungsbedingungen zur Kultivierung eingesetzt [2].

Phytocannabinoide sind Terpenphenole, die aus einem Diphenol- und einem Monoterpenanteil bestehen. Insgesamt wurden bislang über 120 Cannabinoide aus der Cannabispflanze isoliert. Der Hauptbiosyntheseweg (- Abb. 1) erfolgt aus der Olivetolsäure (OLS), die über den Polyketidweg durch schrittweise Kondensation von 3 Malonyl-KoenzymA-Molekülen mit Hexanoyl-Koenzym A gebildet wird [3]. Das Monoterpen Geranyldiphosphat (GPP) alkyliert die OLS über eine spezifische Prenyltransferase zu dem wichtigsten metabolischen Zwischenprodukt, der Cannabigerolsäure (CBGS) [4]. Entsprechende Oxidoreduktasen ((-)trans-Tetrahydrocannabinolsäure(THCS)Synthase, Cannabidiolsäure(CBDS)-Synthase, Cannabichromensäure(CBCS)-Synthase) setzen CBGS weiter zu delta9-Tetrahydrocannabinolsäure (delta9-THCS), Cannabidiolsäure (CBDS) oder Cannabichromensäure (CBCS) um [5-7]. Die Cannabinoidsäuren werden in geringem Ausmaß bereits in der Pflanze durch Licht oder Wärme zu den neutralen, pharmakologisch wirksamen Cannabinoiden delta9Tetrahydrocannabinol (delta9-THC), CBD und Cannabichromen (CBC) decarboxyliert $[8,9]$. Die weitere Decarboxylierung erfolgt durch Erhitzen beim Cannabiskonsum.

Durch die weit verbreitete Hybridisierung bzw. Kreuzung verschiedener geneti- 
scher Linien im legalen wie auch illegalen Anbau entsteht eine Vielzahl von „Cannabissorten", die sich hinsichtlich ihrer Sekundärmetaboliten, insbesondere der beiden hauptsächlich gebildeten Phytocannabinoide delta9-THC und CBD unterscheiden. Weiblen et al. [10] untersuchten die Expression der Cannabinoidsynthasegene und erkannten eine nichtfunktionierende CBDSSynthase in delta9-THC-reichen und CBDarmen Cannabispflanzen. Kojoma et al. [11] untersuchten das Gen der THCS-Synthase von delta9-THC-reichen bzw. CBDreichen Cannabissorten und identifizierten einen spezifischen Marker für das Gen der THC-Synthase von der delta9-THC-reichen Sorte. Somit werden die Cannabisprodukte insbesondere aufgrund ihres delta9-THC-CBD-Verhältnisses in delta9-THCreiche Cannabisprodukte vom "drug type" und CBD-reiche Cannabisprodukte vom "fiber type" (Faserhanf) unterschieden.

Betäubungsmittelrechtlich [12] wird Cannabis in Deutschland als "Marihuana, Pflanzen und Pflanzenteile der zur Gattung Cannabis gehörenden Pflanzen" in der Anlage I des Betäubungsmittelgesetzes (BtMG) als nichtverkehrsfähig und nichtverschreibungsfähig eingestuft. Ausgenommen sind u.a. Samen (sofern sie nicht zum unerlaubten Anbau bestimmt sind), Pflanzen aus zertifiziertem Saatgut oder Pflanzen, deren THC-Gehalt 0,2\% (sog. Faserhanf) nicht übersteigt und deren Verkehr ausschließlich gewerblichen oder wissenschaftlichen Zwecken dient, die einen Missbrauch zu Rauschzwecken ausschließen. Weiterhin ausgenommen sind Cannabis aus einem Anbau zu medizinischen Zwecken unter staatlicher Kontrolle sowie in Zubereitungen, die als Fertigarzneimittel (FA) zugelassen sind (BtMG, Anlage III).

\section{Cannabis als illegale Droge}

In Deutschland hat nach aktuellem epidemiologischen Suchtsurvey (ESA 2018) jeder Vierte im Alter zwischen 18 und 64 Jahren im Leben schon einmal Cannabis konsumiert. Die 12-Monats-Prävalenz des Cannabiskonsums entspricht in Deutschland mit 7,1\% dem europäischen Gesamtmittelwert [13]. Getrocknete weibliche Blüten und Blätter werden als Marihuana und das gewonnene, gepresste Harz als Ha-

Hintergrund: Cannabis ist weltweit immer noch die am häufigsten konsumierte illegale Droge, aber auch der Einsatz von Medizinalcannabis oder auch als Lebens-/Nahrungsergänzungsmittel steigt stetig. Somit sind Kenntnisse über diese verschiedenen Produkte und die Komplexität der rechtlichen Einordnung von Cannabis für die Rechtsmedizin und die forensische Toxikologie von großer Relevanz.

Fragestellung: Ziel der Arbeit ist es, einen Überblick über aktuelle Trends des Cannabiskonsums zu geben und hierbei die verschiedenen Cannabisprodukte darzustellen sowie diese rechtlich einzuordnen.

Material und Methode: Für diese Übersichtarbeit wurde eine Literaturrecherche zu den verschiedenen Cannabisprodukten und ihrer rechtlichen Einordnung durchgeführt.

Ergebnisse: Beim Konsum von Tetrahydrocannabinol(THC)-reichem Cannabis zu Rauschzwecken ist ein Trend hin zu immer höheren THC-Gehalten im Pflanzenmaterial und zusätzlich zu intensiven Konsumformen wie dem "dabbing" von Butan-HaschischÖl zu erkennen. Seit der Betäubungsmittelgesetzesänderung vom 10.03.2017 werden auch Cannabisblüten und -extrakte mit unterschiedlichen THC- bzw. Cannabidiol(CBD)Gehalten auf Betäubungsmittelrezept stark zunehmend verordnet, einhergehend mit einer Steigerung der THC-Höchstverschreibungsmenge. Ein weiterer Trend besteht in dem Konsum von sogenannten CBD-Lifestyle-Produkten, die als Arzneimittel seit 2016 verschreibungspflichtig sind und als Lebensmittel nach Novel Food-Verordnung jeweils einzeln zu prüfen sind, ob sie als zulassungsbedürftiges neuartiges Lebensmittel einzustufen sind.

Schlussfolgerungen: Insgesamt ergibt sich für die forensisch-chemische sowie forensisch-toxikologische Beurteilung eine zunehmende Komplexität der potenziell konsumierten THC-haltigen Produkte, deren wissenschaftliche Untersuchung sowohl zur möglichen Differenzierbarkeit des Cannabismaterials als auch von biologischen Matrices nach Konsum verschiedener Cannabisprodukte notwendig macht.

\section{Schlüsselwörter}

THC $\cdot$ Medizinalcannabis $\cdot$ CBD-Hanf $\cdot$ BtMG $\cdot$ AMG

schisch bezeichnet. Marihuana und Haschisch werden hauptsächlich, mit Tabak gemischt, geraucht (Joint) und seltener oral in Form fettreicher Lebensmittel (z. B. Haschischkekse) konsumiert. Bei Rauchkonsum sind maximale delta9-THC-Plasma-Spiegel unmittelbar (Minuten) nach Beginn des Rauchens erreicht, wohingegen bei oraler Aufnahme die Resorption je nach Magenfüllzustand langsamer (Stunden) erfolgt. ElSoley et al. untersuchten den Wirkstoffgehalt an psychotropen delta9-THC in beschlagnahmtem Marihuana in Europa sowie in den USA und stellten einen Anstieg der delta9-THC-Konzentrationen von durchschnittlich ca. $4 \%$ im Jahr 1995 auf ca. 9\% im Jahr 2008 und auf ca. $17 \%$ im Jahr 2017 fest. Somithat sich bei einem durchschnittlichen Cannabis-Joint mit ca. 200 mg Marihuana oder Haschisch, gemischt mit Tabak [14], die konsumierte delta9-THC-Menge von ca. $8 \mathrm{mg}$ im Jahr 1995 auf $34 \mathrm{mg}$ in Jahr 2017 ungefähr vervierfacht. Zudem stellten die Autoren eine Zunahme beschlagnahmter Haschischölproben fest, die durchschnittlich in ihrer THC-Konzentration von 0,5-4,7\% im Jahr 2008 auf 6,7-55,7\% im Jahr 2017 stiegen $[15,16]$. Der Trend zu immer potenteren Cannabisprodukten zeigt sich in sog. Butan-Haschisch-Ölen (BHO) oder Cannabisextrakten mit anderen abdampfbaren Lösungsmitteln, die THC-Konzentrationen über $60 \%$ aufweisen und durch extrem schnelles Verdampfen auf einem Metall (z. B. Titannagel) über eine Wasserpfeife inhaliert werden, "dabbing". Durch die Konsumform "dabbing" wurde durch Hädener et al. [17] eine vollständige Decarboxylierung von THCS und CBDS zu THC bzw. CBD festgestellt. Es resultiert eine hohe delta9-THC-Verfügbarkeit bei schneller Anflutung, die durch klassisches Rauchen nicht erreicht werden kann. Durch die Zunahme des psychoaktiven Wirkstoffs delta9-THC im Cannabismaterial und durch die Konsumform "dabbing" wird die psychotrope Wirkung verstärkt, und die Gefahr gesundheitlicher Risiken nimmt zu. Die ESA-Studie führt eine Cannabisabhängigkeit von 0,6\% der Studienteilnehmer an und verweist auf den Zusammenhang zwischen Cannabis- 


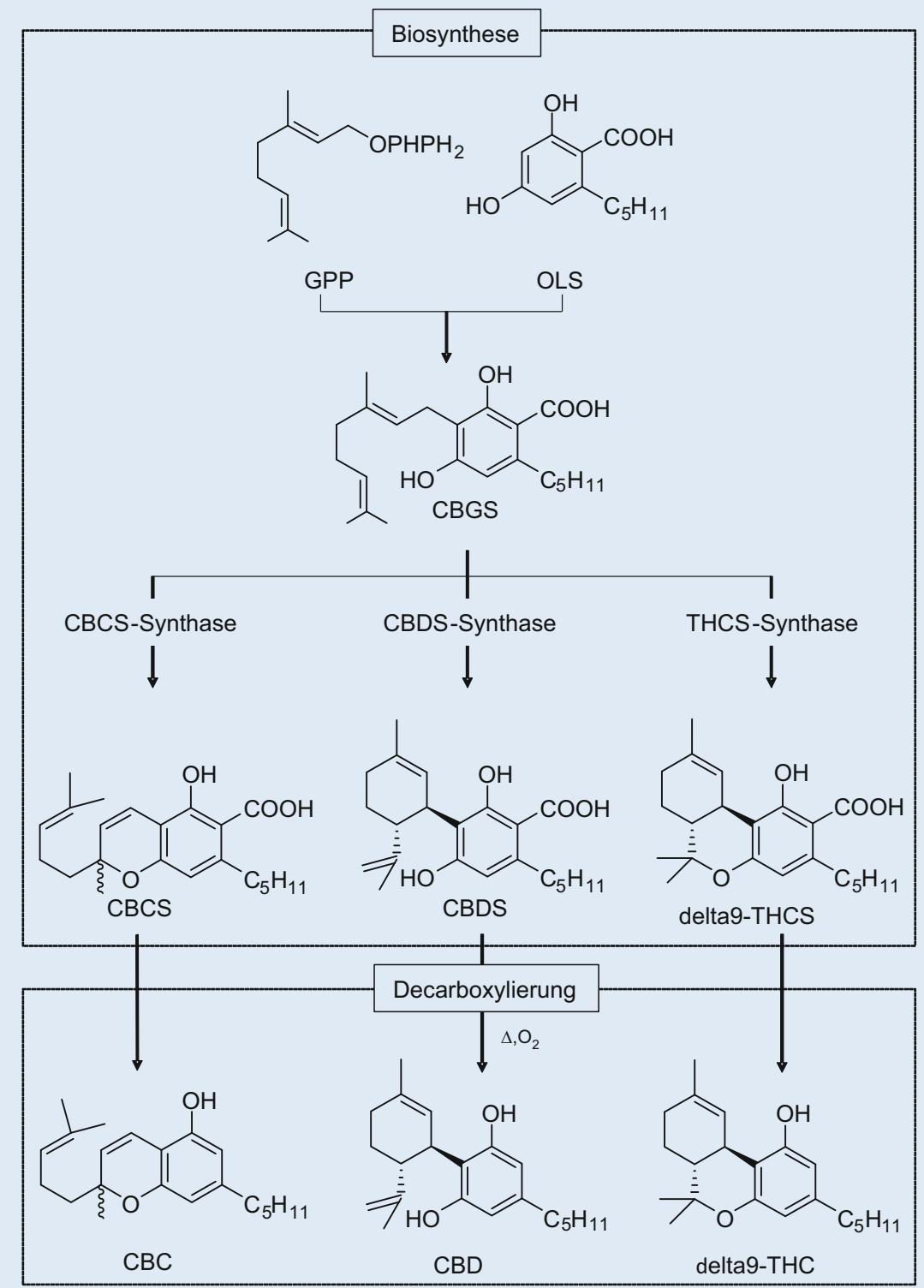

Abb. 1 \ Biosynthese der Phytocannabinoide Cannabichromen (CBC), Cannabidiol (CBD) und delta9Tetrahydrocannabinol (delta9-THC) aus Geranyldiphosphat (GPP) und Olivetolsäure (OLS) über die Cannabigerolsäure (CBGS) zu den jeweiligen Säuren Cannabichromensäure (CBCS), Cannabidiolsäure (CBDS) und delta9-Tetrahydrocannabinolsäure (delta9-THCS)

konsum und Angststörungen, Depressionen sowie bipolaren Störungen [13].

\section{Cannabis als Arzneimittel}

Cannabinoidhaltige Arzneimittel stehen nicht erst seit der Betäubungsmitteländerungsverodnung (BtMÄndV) 2017 für die Therapie zur Verfügung. Bereits seit 1984 ist Nabilon (Canemes ${ }^{\circledR}$ AOP Orphan Pharmaceuticals $\mathrm{GmbH}$, Ismaning, Deutschland), ein vollsynthetisches THCAnalogon (• Abb. 2), als reines Wirkstoff-

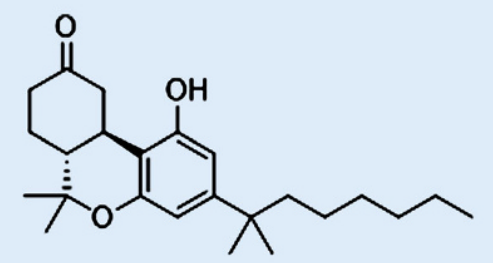

Abb. 2 \ Nabilon

produziert. Es enthält nach Forderung des Deutscher Arzneimittel-Codex (DAC) [18] 95-100\% delta9-THC. Dronabinol ist ein Rezepturarzneimittel, das als Appetitstimulans, als Antiemetikum im Rahmen von Chemotherapie, als Muskelrelaxans und als Analgetikum indiziert ist. Dronabinol kann oral (in Form von Kapseln, Öl) und seit 2017 inhalativ (ethanolische Lösung) mit einer Höchstverschreibungsmenge (HVM) gemäß § 2 BetäubungsmittelVerschreibungsverordnung (BtMVV) von $500 \mathrm{mg}$ delta9-THC/30 Tage $=$ ca. $16 \mathrm{mg}$ delta9-THC/Tag verordnet werden. Als FA ist Dronabinol in den USA unter dem Handelsnamen Marino ${ }^{\circledR}$ zugelassen und kann gemäß $§ 73$ Abs. 3 Arzneimittelgesetz (AMG) importiert werden.

Neben den reinen Wirkstoffpräparaten Dronabinol und Canemes ${ }^{\circledR}$ ist seit 2011 Sativex ${ }^{\circledR}$-Mundspray (GW Pharma (International) B.V., Amersfoort, Niederlande) als erstes in Deutschland zugelassenes cannabinoidhaltiges FA zur Behandlung der multiplen Sklerose verordnungsfähig. Das Mundspray enthält einen Dickextrakt, der durch Auszug aus Blüten und Blättern von Cannabis sativa (THC-Chemotyp bzw. CBD-Chemotyp) mithilfe von flüssigem Kohlendioxid gewonnen wird. Ein Sprühstoß enthält $2,7 \mathrm{mg}$ delta9THC und 2,5 mg CBD, wobei als Maximaldosis 12 Sprühstöße/Tag angegeben werden. Die Verschreibungshöchstmenge beträgt gemäß § 2 BtMVV 1000 mg delta9$\mathrm{THC} / 30$ Tage $=$ ca. $33 \mathrm{mg}$ delta9- $\mathrm{THC} / \mathrm{Tag}$. Somit hat sich die delta9-THC-HVM von Dronabinol mit ca. $16 \mathrm{mg}$ delta9-THC/Tag aus dem Jahr 1998 zu Sativex ${ }^{\circledR}$ mit ca. $33 \mathrm{mg}$ delta9-THC/Tag aus dem Jahr 2011 verdoppelt.

Mit der oben bereits genannten BtMÄndV vom 10.03.2017 ist Cannabis in Form von getrockneten Blüten oder Extrakten in standardisierter Qualität für 
Tab. 1 Übersicht der verschiedenen Cannabisarzneimittel

\begin{tabular}{|c|c|c|c|}
\hline Präparat & Wirkstoff & Darreichungsform & HVM gemäß § 2 BtMVV \\
\hline Canemes $^{\circledR}$ & Nabilon & FA 1-mg-Kapseln (orale Anwendung) & $\begin{array}{l}\text { Keine Angabe; } 180 \mathrm{mg} / 30 \text { Tage (Fachinfor- } \\
\text { mation) }=6 \mathrm{mg} / \mathrm{Tag}\end{array}$ \\
\hline Dronabinol & delta9-THC & $\begin{array}{l}\text { NRF } \\
\text { Oral als Kapseln oder ölige Tropfen } \\
\text { Inhalativ als ethanolische Lösung }\end{array}$ & $500 \mathrm{mg}$ THC $/ 30$ Tage $=c a .16 \mathrm{mg} / \mathrm{Tag}$ \\
\hline Sativex $^{\circledR}$ & Nabiximols (insb. THC/CBD) & $\begin{array}{l}\text { FA: Dickextrakt aus Cannabisblüten und } \\
\text {-blättern (Mundspray) }\end{array}$ & $1000 \mathrm{mg}$ THC$/ 30$ Tage $=c a .33 \mathrm{mg} / \mathrm{Tag}$ \\
\hline Cannabisblüten & $\begin{array}{l}\text { Phytocannabinoide } \\
\text { (insb. THC/CBD) }\end{array}$ & $\begin{array}{l}\text { Delta-9-THC-reiche (bis zu ca. } 25 \% \text { delta9- } \\
\text { THC) bzw. CBD-reiche (bis zu ca. } 15 \% \text { CBD) } \\
\text { Blüten zur oralen oder zur inhalativen } \\
\text { Anwendung }\end{array}$ & $\begin{array}{l}100.000 \mathrm{mg} / 30 \text { Tage (unabhängig vom } \\
\text { THC-Gehalt), d. h. ca. } 33 \mathrm{mg} / \mathrm{Tag} \text { (bei } 1 \% \\
\text { delta9-THC-Gehalt) und ca. } 830 \mathrm{mg} / \mathrm{Tag} \\
\text { (bei } 25 \% \text { delta-9-THC-Gehalt) }\end{array}$ \\
\hline \multirow{2}{*}{$\begin{array}{l}\text { Vollspektrum- } \\
\text { Cannabis- } \\
\text { extrakte }\end{array}$} & \multirow[t]{2}{*}{$\begin{array}{l}\text { Phytocannabinoide } \\
\text { (insb. THC/CBD) }\end{array}$} & $\begin{array}{l}\text { NRF: ölige Cannabisölharzlösung, } 25 \mathrm{mg} / \mathrm{ml} \\
\text { Dronabinol (orale Anwendung) }\end{array}$ & \multirow[t]{2}{*}{$1000 \mathrm{mg}$ THC $/ 30$ Tage $=c a .33 \mathrm{mg} / \mathrm{Tag}$} \\
\hline & & $\begin{array}{l}\text { Sowie verschiedene Extrakte mit variieren- } \\
\text { den THC-/CBD-Gehalten, z. B. Tilray }{ }^{\circledR} \text { oder } \\
\text { Aurora }{ }^{\circledR} \text { bis zu } 50 \mathrm{mg} / \mathrm{ml} \mathrm{THC} \text { und } 20 \mathrm{mg} \\
\mathrm{CBD} / \mathrm{ml} \text { (orale Anwendung) }\end{array}$ & \\
\hline CBD & CBD & $\begin{array}{l}\text { NRF 22.10: ölige CBD-Lösung } 50 \mathrm{mg} \text { oder } \\
100 \mathrm{mg} / \mathrm{ml} \text { (orale Anwendung) }\end{array}$ & \multirow[t]{2}{*}{ Kein BtM, verschreibungspflichtig } \\
\hline Epidyolex $^{\circledR}$ & CBD & $\begin{array}{l}\text { FA: } 100 \mathrm{mg} / \mathrm{ml} \mathrm{CBD-Lösung} \mathrm{(orale} \mathrm{Anwen-} \\
\text { dung) }\end{array}$ & \\
\hline
\end{tabular}

Patienten mit „schwerwiegender Erkrankung" als Betäubungsmittel verordnungsfähig. Voraussetzung zur Verordnung ist, dass eine allgemein anerkannte, dem medizinischen Standard entsprechende Leistung nicht zur Verfügung steht, oder dass eine nicht ganz entfernt liegende Aussicht auf eine spürbare positive Einwirkung auf den Krankheitsverlauf oder auf schwerwiegende Symptome besteht (§ 31 Abs. 6 SGB V). Die Verordnung kann auf Betäubungsmittelrezept durch Ärzte aller Fachrichtungen, ausgenommen Zahn- und Tierärzte, erfolgen. Die Versorgung mit Cannabisprodukten erfolgte zunächst über Importe, z.B. aus Holland (Bedrocan ${ }^{\circledR}$, Veendam, Niederlande) oder Kanada (Aurora ${ }^{\circledR}$ Edmonton, Kanada). In Deutschland wurde die Cannabisagentur, angesiedelt an das Bundesinstitut für Arzneimittel und Medizinprodukte (BfArM), gegründet, welche Anbau, Ernte, Verarbeitung, Qualitätsprüfung, Lagerung sowie Verpackung des für medizinische Zwecke genutzten Cannabis kontrolliert. Es wird nur Cannabis verwendet, das entsprechend den Vorgaben der "Guten Praxis für die Sammlung und den Anbau von Arzneipflanzen" (Good Agricultural and Collection Practice, GACP) angebaut wurde. Zudem muss es den Vorgaben der Monografie "Cannabisblüten“ des
Deutsches Arzneibuch (DAB) [19] mit mindestens $90 \%$ und höchstens 110\% der deklarierten Cannabinoide, berechnet als THC und CBD, entsprechen [20]. Die Cannabisagentur hat 2019 die Unternehmen Aurora Productions $\mathrm{GmbH}$, Aphria Deutschland $\mathrm{GmbH}$ und DEMECAN GmbH für Anbau, Ernte und Verarbeitung von Cannabis zu medizinischen Zwecken über insgesamt $10.400 \mathrm{~kg}$ für 4 Jahre beauftragt [21]. Die Logistik des Vertriebs wird durch das Unternehmen Cansativa $\mathrm{GmbH}$ durchgeführt. Seit dem 07.07.2021 ist nun auch Cannabis für medizinische Zwecke aus deutschem Anbau über die Cannabisagentur von Apotheken bestellbar [22].

Cannabisblüten sind mit einer HVM, unabhängig vom THC-Gehalt, von $100.000 \mathrm{mg} / 30$ Tage gemäß § 2 BtMVV als Betäubungsmittel verordnungsfähig [23]. Bei CBD-reichem Cannabis mit nur ca. 1\%igem delta9-THC-Gehalt resultiert eine maximale Menge von ca. $33 \mathrm{mg}$ delta9-THC/Tag, was der HVM anderer Cannabisprodukte entspricht. Bei THC-reichen Cannabisblüten mit ca. $25 \%$ delta9THC-Gehalt ist rechnerisch eine maximale delta9-THC-Menge von ca. $830 \mathrm{mg} / \mathrm{Tag}$ zu erreichen. Diese delta9-THC-Menge entspräche einer Menge von mehr als
20 Joints/Tag, wenn man eine Menge von ca. $35 \mathrm{mg}$ delta9-THC/Joint annimmt [14].

Neben den Cannabisblüten stehen vermehrt auch sog. Vollspektrum-Cannabisextrakte zur Verfügung. Durch Interaktion der verschiedenen Cannabisinhaltsstoffe wird eine bessere Wirkung erzielt als ggf. bei Einzelwirkstoffen, was als Entourage-Effekt bezeichnet wird. In Neues Rezeptur-Formularium (NRF) [18] wird eine auf $25 \mathrm{mg} / \mathrm{ml}$ Dronabinol standardisierte ölige Cannabisölharzlösung (NRF 22.11) aufgeführt. Des Weiteren stehen Vollspektrum-Cannabisextrakte mit unterschiedlichen, standardisierten Gehalten an delta9THC und CBD, beispielsweise von der Fa Tilray ${ }^{\circledR}$ (Tilray Deutschland GmbH, Berlin, Deutschland), zur Verfügung. Diese sind in Deutschland als Arzneimittel zur Herstellung von Rezepturarzneimitteln zugelassen. Das bedeutet, dass der letzte Herstellungsschritt vor Abgabe der Produkte an den Patienten in der Apotheke stattfindet. Die HVM ist $1000 \mathrm{mg}$ delta9-THC/30 Tage $=$ ca. $33 \mathrm{mg}$ delta9-THC/Tag.

$C B D$ unterliegt als nichtpsychotropes Cannabinoid zwar nicht dem BtMG, ist aber als CBD-haltiges Arzneimittel seit 2016 ohne Eingrenzung von Dosis oder Verabreichungsform verschreibungspflichtig. Seit 2015 wird im NRF eine ölige Cannabidiollösung zum Einnehmen aufgeführt, 


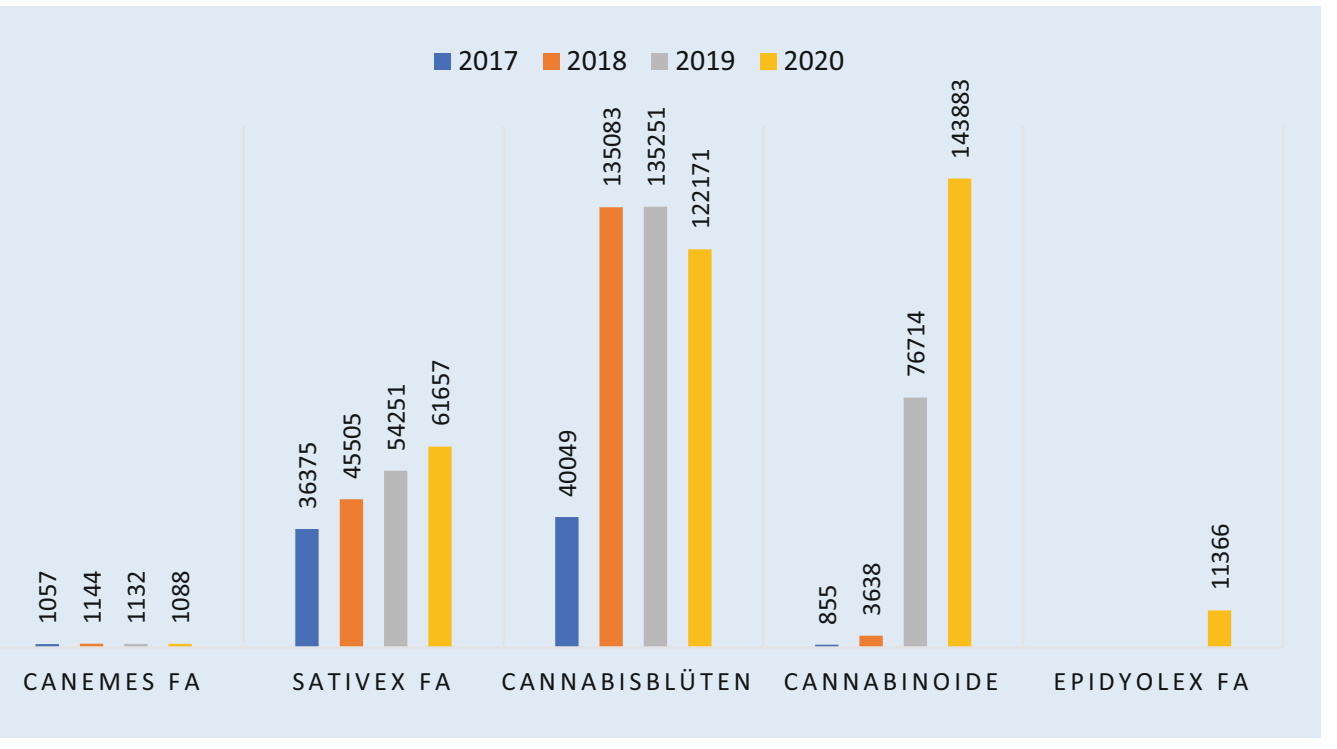

Abb. $3 \triangleleft$ Verordnungen von cannabinoidhaltigen Fertigarzneimitteln (FA) und Zubereitungen (Sonderbeilagen zur GKV-Arzneimittelschnellinformation für Deutschland nach $\S 84$ Abs. 5 SGB V) [25-27]

die beim Dravet-Syndrom (DS) und beim Lennox-Gastaut-Syndrom (LGS), bei multipler Sklerose und anderen Anwendungsgebieten bei individuell zu stellender Indikation eingesetzt werden kann. Die CBDTagesdosen können vom unteren zweistelligen bis in den mittleren dreistelligen Milligrammbereich gehen.

Seit 2019 ist das FA Epidyolex ${ }^{\circledR}$ (GW Pharma (International) B.V., Amersfoort, Niederlande) in Europa (USA Epidolex ${ }^{\circledR}$ 2018) als orale $100 \mathrm{mg} / \mathrm{ml}$-CBD-Lösung verfügbar. Es wird zusammen mit dem Benzodiazepin Clobazam bei Patienten ab 2 Jahren für die adjuvante Behandlung von Krampfanfällen im Zusammenhang mit dem LGS oder dem DS angewendet. Je nach individuellem klinischen Ansprechen und der Verträglichkeit kann die Dosis bis $20 \mathrm{mg} / \mathrm{kg} \mathrm{KG}$ und Tag betragen [24].

Die verschiedenen Cannabisarzneimittel sind abschließend in 0 Tab. 1 zusammengefasst. Hierbei werden sie in reine Wirkstoffpräparate, Cannabisextrakte bzw. Cannabisblüten mit standardisierten THC/CBD-Gehalten differenziert sowie in Rezepturarzneimittel des NRF und FA eingeteilt. Zusätzlich werden die Dosierungen bzw. die HVM gemäß § 2 BtMVV angegeben.

In - Abb. 3 sind die Verordnungen cannabinoidhaltiger FA und Zubereitungen der Jahre 2017 bis 2020 dargestellt [25-27]. Das seit 1984 zur Verfügung stehende FA Canemes ${ }^{\circledR}$ (Nabilon) wird unverändert selten (ca. 1000/Jahr) verordnet. Das seit 2011 zur Verfügung stehende FA
Sativex ${ }^{\circledR}$ wird wesentlich häufiger verordnet (ca. 55.000/Jahr) und zeigt eine stetige geringe Zunahme der Verordnungszahlen. Cannabisblüten starten 2017 mit ca. 40.000 Verordnungen, die 2018 auf ca. 130.000 Verordnungen hochschnellen und sich dann weitestgehend konstant bis 2020 halten. Die Gruppe der cannabinoidhaltigen Arzneimittel zeigt einen starken Anstieg von 3638 Verordnungen im Jahr 2018 auf 143.883 Verordnungen im Jahr 2020. Der Report des Spitzenverbandes Bund der Krankenkassen (GKVSpitzenverband) [25-27] lässt leider keine Differenzierung der Cannabinoide in beispielsweise Dronabinol, CBD oder Vollspektrum-Cannabisextrakte zu. Nur das CBD-FA Epidyolex ${ }^{\circledR}$, mit Erstzulassung 2019, wird differenziert in dem Bericht genannt. Die GKV-Berichte verdeutlichen die Relevanz der Cannabisarzneimittel, insbesondere der seit 2017 verordnungsfähigen Cannabisblüten und Cannabisextrakte.

\section{Cannabis als Lebensmittel und Nahrungsergänzungsmittel}

Im Jahr 1996 wurde der Anbau von Faserhanf, dessen THC-Gehalt 0,2\% nicht übersteigt und der ausschließlich gewerblichen oder wissenschaftlichen Zwecken dient, die einen Missbrauch zu Rauschzwecken ausschließen, legalisiert. Neben der industriellen Nutzung des Faserhanfs wurden vielfältige Lebensmittel aus Hanfsamen, z.B. Hanfsamenöl oder Mehl, hergestellt [28]. Ein neuer Trend geht hin zu CBD-Produkten als sog. LifestyleProdukte, die entweder auf Hanfextrakten zumeist aus der Gesamtpflanze, inkl. Blüten, basieren, oder sog. CBD-Blüten, die als solche gehandelt oder als Zutat eingesetzt werden. Diese werden als Nahrungsergänzungsmittel ohne gesundheitsbezogene Werbeaussage in Drogerien und im Internet vermarket und unterliegen damit nicht dem Arzneimittelgesetz bzw. der Verschreibungspflicht. In der Arbeit des wissenschaftlichen Dienstes 5: „Wirtschaft und Verkehr, Ernährung, Landwirtschaft und Verbraucherschutz des Deutschen Bundestages" vom 22.07.2019 wird zur Verkehrsfähigkeit von CBD-haltigen Lebensmitteln resümiert, dass das $C B D$ als neuartiges und damit zulassungsbedürftiges Lebensmittel in die Novel-FoodVerordnung (NFV, (EU) 2015/2283) einzuordnen sei. Ob ein konkretes Produkt allerdings als zulassungsbedürftiges neuartiges Lebensmittel einzustufen ist oder nicht, sei stets eine Einzelfallprüfung [29, 30]. Nach Empfehlung des Bundesinstituts für gesundheitlichen Verbraucherschutz und Veterinärmedizin (BgVV) aus dem Jahr 2000 sollte die tägliche Aufnahmemenge von delta9-THC, inklusive delta9THCS, mit hanfhaltigen Lebensmitteln $1-2 \mu \mathrm{g} / \mathrm{kg}$ KG nicht überschreiten. Unter Einbeziehung dieser Empfehlung und unter der Annahme einer täglichen Aufnahme durchschnittlicher Verzehrsmengen hanfhaltiger Produkte wurden folgende Richtwerte für Lebensmittel abgeleitet: $5 \mu \mathrm{g} / \mathrm{kg} \mathrm{KG}$ für nichtalkoholische und al- 
koholische Getränke; $5000 \mu \mathrm{g} / \mathrm{kg}$ KG für Speiseöle (auch für kosmetische Produkte) und $150 \mu \mathrm{g} / \mathrm{kg}$ KG für alle anderen Lebensmitteln [31]. Seit dem Aufkommen der CBD-Produkte werden durch die Lebensmittelüberwachungsbehörden extrem überhöhte THC-Gehalte berichtet [32, 33]. Lachenmeier et al. [32] stellten bei $43 \%$ der untersuchten Produkte $(n=28)$ unter Berücksichtigung der Tagesverzehrsempfehlung fest, dass eine delta9-THC-Tagesdosis von mehr als $1 \mathrm{mg}$ aufgenommen würde, die in Einzelfällen sogar bis zu $30 \mathrm{mg}$ erreiche also eine Dosis, die annähernd einem durchschnittlichen Joint entspricht. Das Bundesinstitut für Risikobewertung (BfR) empfiehlt in der Stellungnahme 006/2021 vom 17.02.2021 eine akute Referenzdosis (ARfD) von $1 \mu \mathrm{g}$ delta9-THC(S)/kg KG als Grundlage zur Beurteilung hanfhaltiger Lebensmittel. Hierzu sollte jedes Produkt anhand gemessener THC-Gehalte und geschätzter Verzehrsmenge geprüft werden [34]. Des Weiteren wird vermehrt CBDHanf sichergestellt, der mit synthetischen Cannabinoiden versetzt ist [35]. Wie die halbsynthetische Herstellung von Dronabinol zeigt und wie in einem US-Patent [36] beschrieben ist, kann CBD chemisch zu dem psychotrop wirkenden delta9THC umgesetzt werden. Kiselak et al. [37] beschreiben die säurekatalysierte Isomerisierung von $\mathrm{CBD}$ zu u.a. delta9-THC durch allgemein zur Verfügung stehende Reagenzien, wie z.B. $30 \%$ ige Salzsäure, 5,4\%ige Essigsäure. Mascal et al. [38] führen daher an, dass bei unkontrollierter CBD-Verfügbarkeit die Gefahr einer chemischen Synthese besteht, analog zu dem "Meth-lab"-Phänomen, der Umsetzung von Pseudoephedrin zu Methamphetamin.

\section{Cannabis in der forensisch- toxikologischen Beurteilung}

Die aufgezeigten aktuellen Trends des Cannabiskonsums zeigen, dass die Cannabispflanze umfangreich, in vielfältiger Weise und zu verschiedensten Zwecken konsumiert wird. Zu Rauschzwecken wird bevorzugt das psychotrop wirksame delta9-THC-reiche Cannabis inhalativ als Joint geraucht, mit einer konsumierten durchschnittlichen delta9-THC-Menge von ca. $35 \mathrm{mg}$. Zu medizinischen Zwecken stehen seit 2017 nicht mehr nur cannabishaltige Fertigarzneimittel oder Rezepturarzneimittel mit einer HVM bis zu $33 \mathrm{mg}$ delta9-THC pro Tag zur Verfügung, sondern auch Cannabisblüten mit unterschiedlichen Gehalten an delta9-THC und CBD, mit einer HVM bis zu ca. $830 \mathrm{mg}$ delta9-THC/Tag. Medizinische Cannabisblüten lassen sich derzeit weder optisch noch durch forensisch-chemische Analyse sicher von illegalen Cannabisblüten unterscheiden. Zudem eignen sich medizinische Cannabisblüten prinzipiell auch zu Rauschzwecken. So nennen Geile et al. [39] als eine von 3 Patientengruppen die Konsumenten, die eine Missbrauchsvorgeschichte haben und eine Cannabisverschreibung aus medizinischen Gründen anstreben, um ihren illegalen Konsum zu legalisieren. Verkehrsmedizinisch liegt nach § 24a Straßenverkehrsgesetz (StVG) nämlich u. a. eine Ordnungswidrigkeit vor, wenn im Straßenverkehr ein Kraftfahrzeug von einer Person geführt wird, die unter dem berauschendem Mittel Cannabis, der Substanz THC, stand. Ausgenommen ist eine Ahndung, wenn die Substanz aus der bestimmungsgemäßen Einnahme eines für einen konkreten Krankheitsfall verschriebenen Arzneimittels herrührt. Daher ist eine verantwortungsvolle Verschreibungspraxis von erfahrenen, geschulten Ärzten notwendig, um diese Cannabispatienten zu erkennen und damit auch Schaden von dem verantwortungsbewussten Cannabispatienten abzuhalten. Wie bei anderen zentralwirkenden Medikamenten ist weiterhin zu beachten, dass in der Einstellungsphase bis zur stabilen symptomarmen Therapiephase bei gleichbleibender regelmäßiger Medikation das Leistungsbild verlässlich hinsichtlich einer möglichen Fahrerlaubnis einzuhalten ist. Ob bei stabiler Dosierung die Teilnahme am Straßenverkehr möglich ist, muss in jedem Einzelfall nach Rücksprache mit dem Patienten vom Arzt entschieden werden [40]. Die Fahrerlaubnisbehörde kann bei Bekanntwerden einer Verordnung von Cannabisarzneimitteln die Beibringung eines ärztlichen Gutachtens anordnen, um etwa den verantwortungsvollen Umgang des Patienten mit dem Medikament im Zusammenhang mit der Teilnahme am Straßenverkehr abzuklären [40].
Eine weitere Problemstellung der forensisch-toxikologischen Beurteilung ergibt sich aus der Einlassung potenziell legaler cannabishaltiger Lebensmittel oder CBD-Lifestyle-Produkte, die vereinzelt bei Berücksichtigung der Tagesverzehrsempfehlung bis hin zu rauschfähigen delta9THC-Mengen führen können. Hier sind je nach zeitlichem Abstand zwischen Konsum und Probennahme positive Cannabinoidnachweise im Blut bzw. im Urin möglich. Auch sind positive Cannabinoidbefunde im Haar nach Verwendung von Hanföl als Haarkosmetik beschrieben [41]. Daher ist bei der Abstinenzvertragsgestaltung eine Aufklärung hinsichtlich des Umgangs cannabishaltiger Produkte notwendig.

\section{Fazit}

- Aus der Cannabispflanze können aufgrund von Hybridisierung/Kreuzung verschiedenste "Cannabissorten" mit variablen Gehalten an Sekundärmetaboliten, insbesondere an Tetrahydrocannabinol (THC) und Cannabidiol (CBD), gewonnen werden.

- Cannabis ist immer noch mit Abstand die am häufigsten konsumierte illegale Droge weltweit, wobei ein Trend zu immer THC-potenteren Cannabisprodukten festzustellen ist.

- Mit der Betäubungsmitteländerungsverordnung (BtMÄndV) vom 10.03.2017 werden zusätzlich zu den Arzneimitteln mit den Wirkstoffen Dronabinol oder Nabilon nunmehr auch Cannabisblüten und -extrakte mit unterschiedlichen delta9-THC-/CBDGehalten auf Betäubungsmittelrezept stark zunehmend verordnet. Seit dem 07.07.2021 ist Cannabis aus deutschem Anbau über die Cannabisagentur von Apotheken bestellbar.

- Die Höchstverschreibungsmenge (HVM) gemäß § 2 Betäubungsmittelverschreibungsverordnung (BtMVV) ist von ca. $16 \mathrm{mg}$ delta9-THC/Tag im Jahr 1998 (Dronabinolrezeptur) auf ca. 33 mg delta9-THC/Tag (Sativex ${ }^{\circledR}$ bzw. Cannabisextrakte) auf bis zu ca. $830 \mathrm{mg}$ delta9-THC/Tag (Cannabisblüte mit ca. $25 \%$ delta9-THC-Gehalt) im Jahr 2017 deutlich angestiegen.

- CBD ist als Arzneimittel seit 2016 verschreibungspflichtig, trotzdem 
ist ein steigender Konsum von CBD-Produkten als Lebensmittel/ Nahrungsergänzungsmittel bzw. als CBD-Hanf zu erkennen, sowie ein Zusetzen von synthetischen Cannabinoiden.

- Somit ergibt sich für die forensischchemische sowie forensisch-toxikologische Beurteilung eine zunehmende Komplexität der potenziell konsumierten THC-/CBD-haltigen Produkte, deren wissenschaftliche Untersuchung sowohl zur möglichen Differenzierbarkeit des Cannabismaterials als auch des Konsums verschiedener Cannabisprodukte in biologischen Matrices notwendig ist.

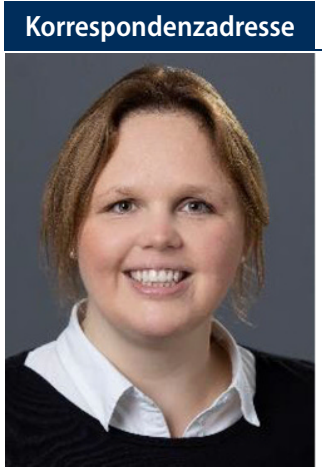

Dr. rer. nat. J. Schürenkamp

Institut für Rechtsmedizin, Forensische Toxikologie, Universitätsklinikum Münster Röntgenstr. 23, 48149 Münster, Deutschland jennifer.schuerenkamp@ukmuenster.de

Danksagung. Die Autorin dankt dem Dekanat der Medizinischen Fakultät der Westfälischen WilhelmsUniversität Münster für die Unterstützung dieses Beitrags (18-003).

\section{Einhaltung ethischer Richtlinien}

Interessenkonflikt. J. Schürenkamp gibt an, dass kein Interessenkonflikt besteht.

Für diesen Beitrag wurden von der Autorin keine Studien an Menschen oder Tieren durchgeführt. Für die aufgeführten Studien gelten die jeweils dort angegebenen ethischen Richtlinien.

Open Access. Dieser Artikel wird unter der Creative Commons Namensnennung 4.0 International Lizenz veröffentlicht, welche die Nutzung, Vervielfältigung, Bearbeitung, Verbreitung und Wiedergabe in jeglichem Medium und Format erlaubt, sofern Sie den/die ursprünglichen Autor(en) und die Quelle ordnungsgemäß nennen, einen Link zur Creative Commons Lizenz beifügen und angeben, ob Änderungen vorgenommen wurden.

Die in diesem Artikel enthaltenen Bilder und sonstiges Drittmaterial unterliegen ebenfalls der genannten Creative Commons Lizenz, sofern sich aus der Abbildungslegende nichts anderes ergibt. Sofern das betreffende Material nicht unter der genannten Creative Commons Lizenz steht und die betreffende Handlung nicht nach gesetzlichen Vorschriften erlaubt ist, ist für die oben aufgeführten Weiterverwendungen des $\mathrm{Ma}$ terials die Einwilligung des jeweiligen Rechteinhabers einzuholen.

Weitere Details zur Lizenz entnehmen Sie bitte der Lizenzinformation auf http://creativecommons.org/ licenses/by/4.0/deed.de.

\section{Literatur}

1. McPartland JM, Guy GW (2017) Models of cannabis taxonomy, cultural bias, and conflicts between scientific and vernacular names. Bot Rev 83(4):327-381. https://doi.org/10.1007/s12229017-9187-0

2. Grotenhermen F (2001) Cannabis und Cannabinoide: Pharmakologie, Toxikologie und therapeutisches Potential, 1. Aufl. Huber, Bern

3. Gagne SJ, Stout JM, Liu E et al (2012) Identification of olivetolic acid cyclase from cannabis sativa reveals a unique catalytic route to plant polyketides. Proc Natl Acad Sci USA 109(31):12811-12816. https://doi.org/10.1073/pnas.1200330109

4. FellermeierM,ZenkMH(1998) Prenylation of olivetolate by a hemp transferase yields cannabigerolic acid, the precursor of tetrahydrocannabinol. FEBS Lett 427(2):283-285. https://doi.org/10.1016/ s0014-5793(98)00450-5

5. Sirikantaramas S, Morimoto S, Shoyama $Y$ et al (2004) The gene controlling marijuana psychoactivity: molecular cloning and heterologous expression of Delta1-tetrahydrocannabinolic acid synthase from cannabis sativa L. J Biol Chem 279(38):39767-39774. https://doi.org/10.1074/ jbc.m403693200

6. Sirikantaramas S, Taura F, Tanaka Y et al (2005) Tetrahydrocannabinolicacidsynthase, theenzyme controlling marijuana psychoactivity, is secreted into the storage cavity of the glandular trichomes. Plant Cell Physiol 46(9):1578-1582. https://doi. org/10.1093/pcp/pci166

7. Taura F, Dono E, Sirikantaramas S et al (2007) Production of Delta(1)-tetrahydrocannabinolic acid by the biosynthetic enzyme secreted from transgenic pichia pastoris. Biochem Biophys Res Commun 361(3):675-680. https://doi.org/10. 1016/j.bbrc.2007.07.079

8. Lange BM, Turner GW (2013) Terpenoid biosynthesis in trichomes - current status and future opportunities. Plant Biotechnol J 11(1):2-22. https://doi.org/10.1111/j.1467-7652.2012.00737. $\mathrm{x}$

9. Happyana N, Agnolet S, Muntendam Ret al (2013) Analysis of cannabinoids in laser-microdissected trichomes of medicinal cannabis sativa using LCMS and cryogenic NMR. Phytochemistry 87:51-59. https://doi.org/10.1016/j.phytochem.2012.11. 001

10. Weiblen GD, Wenger JP, Craft KJ et al (2015) Gene duplication and divergence affecting drug content in cannabis sativa. New Phytol 208(4):1241-1250. https://doi.org/10.1111/nph.13562
11. Kojoma M, Seki H, Yoshida S et al (2006) DNA polymorphisms in the tetrahydrocannabinolic acid (THCA) synthase gene in "drug-type" and "fiber-type" cannabis sativa L. Forensic Sci Int 159(2):132-140. https://doi.org/10.1016/j. forsciint.2005.07.005

12. Gesetz über den Verkehr mit Betäubungsmitteln (Betäubungsmittelgesetz - BtMG), veröffentlicht im BGBI.IS. 1083 vom 12.07.2019

13. Atzendorf J, Rauschert C, Seitz N-N et al (2019) The use of alcohol, tobacco, illegal drugs and medicines. Dtsch Arztebl Int 116(35):577-584. https://doi.org/10.3238/arztebl.2019.0577

14. Europäische Beobachtungsstelle für Drogen und Drogensucht (EMCDDA) (2021) Cannabis drug profile. https://www.emcdda.europa.eu/ publications/drug-profiles/cannabis_en. Zugegriffen:22. Aug. 2021

15. Chandra S, Radwan MM, Majumdar CG et al (2019) New trends in cannabis potency in USA and Europe during the last decade (2008-2017). Eur Arch Psychiatry Clin Neurosci 269(1):5-15. https://doi. org/10.1007/s00406-019-00983-5

16. ElSohly MA, Mehmedic Z, Foster S et al (2016) Changes in cannabis potency over the last 2 decades (1995-2014): analysis of current data in the United States. Biol Psychiatry 79(7):613-619. https://doi.org/10.1016/j.biopsych.2016.01.004

17. Hädener M, Vieten S, Weinmann W et al (2019) A preliminary investigation of lung availability of cannabinoids by smoking marijuana or dabbing $\mathrm{BHO}$ and decarboxylation rate of THC- and CBDacids. Forensic Sci Int 295:207-212. https://doi. org/10.1016/j.forsciint.2018.12.021

18. Deutscher Arzneimittel-Codex/Neues RezepturFormularium (DAC/NRF), Ergänzungsbuch zum Arzneibuch von der DAC/NRF-Kommission und der ABDA - Bundesvereinigung Deutscher Apothekerverbände e.V. (Hrsg.), ISBN: 978-37741-0044-2

19. Deutsches Arzneibuch (DAB) Fortsetzungswerk, 408S., Loseblattwerkin 1 Ordner, am 01.10.2021 in Kraft getreten; ISBN: 978-3-7692-7804-0

20. Bundesinstitut für Arzneimittel und Medizinprodukte (2020) Cannabisagentur. https://www. bfarm.de/DE/Bundesopiumstelle/Cannabis/ Cannabisagentur/_node.html. Zugegriffen: 31. Mai 2020

21. Bundesinstitut für Arzneimittel und Medizinprodukte (2019) Vergabeverfahren erfolgreich abgeschlossen: BfArM erteilt verbliebene vier Zuschläge für Anbau von Cannabis zu medizinischen Zwecken (Nummer 4/19)

22. Deutsche Apotheker Zeitung online (DAZ) (2021) Apotheken können Cannabis beim BfArM kaufen. https://www.deutsche-apotheker-zeitung.de/ news/artikel/2021/07/08/apotheken-koennencannabis-beim-bfarm-kaufen. Zugegriffen: 22. Aug. 2021

23. Kiefer A, Eckert-Lill C, Bussick D et al (2019) Cannabis für medizinische Zwecke und seine ärztliche Verordnung. Bundesgesundheitsblatt Gesundheitsforschung Gesundheitsschutz 62(7):811-817. https://doi.org/10.1007/s00103019-02970-6

24. Fachinformation (2019) Epidyolex $100 \mathrm{mg} / \mathrm{ml}$ Lösung zum Einnehmen, S1-9

25. GKV-Arzneimittel-Schnellinformation (GAmSi) (2021) Sonderbeilage zur GKV-ArzneimittelSchnellinformation für Deutschland. Bruttoumsätze und Verordnungen von cannabinoidhaltigen Fertigarzneimitteln und Zubereitungen von Januar bis Dezember 2020, S1-3 
26. GKV-Arzneimittel-Schnellinformation (GAmSi) (2020) Sonderbeilage zur GKV-ArzneimittelSchnellinformation für Deutschland. Bruttoumsätze und Verordnungen von cannabinoidhaltigen Fertigarzneimitteln und Zubereitungen von Januar bis Dezember 2019,S1-3

27. GKV-Arzneimittel-Schnellinformation (GAmSi) (2019) Sonderbeilage zur GKV-ArzneimittelSchnellinformation für Deutschland. Bruttoumsätze und Verordnungen von cannabinoidhaltigen Fertigarzneimitteln und Zubereitungen von Januar bis Dezember 2018, S1-2

28. Habel S, Sproll C, Teipel J et al (2020) Positive Cannabis-Urintests durch kommerzielle CannabidiolProdukte. Toxichem Krimtech 87(1):10-18

29. Wirtschaftund Verkehr, Ernährung, Landwirtschaft und Verbraucherschutz (2019) Verkehrsfähigkeit von Cannabidiol (CBD)-haltigen Lebensmitteln, S 1-21 (WD 5-3000-065/19)

30. Lachenmeier D, Rajcic de Rezende T et al (2020) Aktuelle Rechtsprechung bestätigt Novel-FoodEinstufung von Hanfextrakten und Cannabidiol (CBD) in Lebensmitteln - Betäubungsmitteleinstufung von Cannabislebensmitteln ist weiterhin unklar. Dtsch Lebensmitt Rundsch 116:111-119. https://doi.org/10.5281/zenodo.3631608.27

31. Bundesinstitut für Risikobewertung (BfR) (2000) BgVV empfiehlt Richtwerte für THC in hanfhaltigen Lebensmitteln. https://www.bfr.bund.de/ de/presseinformation/2000/07/bgvv_empfiehlt_ richtwerte_fuer_thc_tetrahydrocannabinol in_hanfhaltigen_lebensmitteln-884.html.Zugegriffen: 11.Dez. 2021

32. Habel S, Sproll C, Teipel J et al (2020) Positive Cannabis-Urintests durch kommerzielle CannabidiolProdukte. Toxichem Krimtech 87(1):10

33. Bundesinstitut für Risikobewertung (BfR) (2018) Tetrahydrocannabinoidgehalte sind in vielen hanfhaltigen Lebensmitteln zu hoch - gesundheitliche Beeinträchtigungen sind möglich (Stellungnahme Nr.034/2018)

34. Bundesinstitut für Risikobewertung (BfR) (2021) Akute Referenzdosis als Grundlage zur Beurteilung hanfhaltiger Lebensmittel https://doi. org/10.17590/20210217-124105 (Stellungnahme Nr.006/2021)

35. Gemeinsame Pressemitteilung des Bundeskriminalamtes und des Zollkriminalamtes (2021) Gefährliche Entwicklungen auf dem Rauschgiftmarkt - Warnung vor Cannabisprodukten mit synthetischen Cannabinoiden

36. Webster GB, Sarna LP, Mechoulam R (2004) Conversion of CBD to delta8-THC and delta9-THC (US7,399,872 B2)

37. Kiselak TD, Koerber R, Verbeck GF (2020) Synthetic route sourcing of illicit at home cannabidiol (CBD) isomerization to psychoactive cannabinoids using ion mobility-coupled-LC-MS/MS. Forensic Sci Int 308:110173. https://doi.org/10.1016/j.forsciint. 2020.110173

38. Mascal M, Hafezi N, Wang D et al (2019) Synthetic, non-intoxicating 8,9-dihydrocannabidiol for the mitigation of seizures. Sci Rep 9(1):7778. https:// doi.org/10.1038/s41598-019-44056-y

39. Geile J, Graw Metal (2021) Medizinisches Cannabis im Straßenverkehr. Blutalkohol 58(1):1-11

40. Mußhoff F, Graw M (2019) Cannabisblüten als Medikation und Teilnahme am Straßenverkehr - Beispiele für Patiententypen. Blutalkohol 56(2):73-83

41. Paul R, Williams R, Hodson V et al (2019) Detection of cannabinoids in hair after cosmetic application of hemp oil. Sci Rep 2019(9):2582. https://doi.org/ 10.1038/s41598-019-39609-0

\section{Trends in cannabis use}

Background: Cannabis is still the most commonly used illicit drug worldwide. Additionally, the use of medicinal cannabis or as a food/nutritional supplement is increasing. Thus, knowledge about these different products and the complexity of the legal classification of cannabis is of great interest for forensic medicine and toxicology. Objective: The aim of this manuscript is to provide an overview of current trends in cannabis use by presenting different cannabis products and to discuss their legal status.

Material and methods: For this review, a literature search was conducted on the various cannabis products and their legal classification.

Results: For cannabis as an illicit drug, there is a trend towards increasing tetrahydrocannabinol (THC) contents in the plant material and towards more intensive forms of consumption, such as "dabbing" of butane hash oil. With the BtMG amendment of 10.03.2017 cannabis flowers and extracts with different THC-CBD (cannabidiol) contens are now increasingly being prescribed on narcotics prescriptions. This is combined with an increase in the maximum prescription quantity. Another trend is the consumption of so-called CBD lifestyle products, which as medical products have been subjected to prescription since 2016. According to the Novel Food Regulation a CBD food must be individually assessed to determine whether it qualifies as a novel food requiring authorization.

Conclusion: Overall, for forensic chemical as well as forensic toxicological assessment, there is an increasing complexity of potentially consumed THC-containing products, the scientific investigation of which is necessary both for the possible differentiability of cannabis material as well as cannabis consumption of different cannabis products from biological samples.

\section{Keywords}

THC $\cdot$ Medical cannabis · CBD hemp · BtMG · AMG 